\title{
Syphilis: Re-emergence of an old foe
}

\author{
Lola V. Stamm \\ Department of Epidemiology, Gillings School of Global Public Health, University of North Carolina at Chapel Hill, Chapel Hill, NC \\ 27599-7435. \\ * Corresponding Author: \\ Lola V. Stamm, Tel: +919-966-3809; E-mail: Istamm@email.unc.edu
}

\begin{abstract}
Syphilis is caused by infection with Treponema pallidum subsp. pallidum, a not-yet-cultivable spiral-shaped bacterium that is usually transmitted by sexual contact with an infected partner or by an infected pregnant woman to her fetus. There is no vaccine to prevent syphilis. Diagnosis and treatment of infected individuals and their contacts is key to syphilis control programs that also include sex education and promotion of condom use to prevent infection. Untreated syphilis can progress through four stages: primary (chancre, regional lymphadenopathy), secondary (disseminated skin eruptions, generalized lymphadenopathy), latent (decreased re-occurrence of secondary stage manifestations, absence of symptoms), and tertiary (gummas, cardiovascular syphilis and late neurological symptoms). The primary and secondary stages are the most infectious. WHO estimates that each year 11 million new cases of syphilis occur globally among adults aged 15-49 years. Syphilis has re-emerged in several regions including North America, Western Europe, China and Australia. Host-associated factors that drive the reemergence and spread of syphilis include high-risk sexual activity, migration and travel, and economic and social changes that limit access to health care. Early, uncomplicated syphilis is curable with a single intramuscular injection of benzathine penicillin G (BPG), the first line drug for all stages of syphilis. Emergence of macrolide-resistant $T$. pallidum has essentially precluded the empirical use of azithromycin as a second-line drug for treatment of syphilis. Virulence attributes of $T$. pallidum are poorly understood. Genomic and proteomic studies have provided some new information concerning how this spirochete may evade host defense mechanisms to persist for long periods in the host.
\end{abstract}

doi: $10.15698 /$ mic2016.09.523

Received originally: 10.09.2015;

in revised form: 08.01.2016,

Accepted 11.01.2016,

Published 27.06.2016.

Keywords: Syphilis, Treponema pallidum, spirochete, macrolide resistance, re-emerging infectious disease, sexually transmitted infection.
Abbreviations:
$B F P$ - biological false positive $B P G$ - benzathine penicillin $G$, $C D C$ - Center for Disease Control, IM-intramuscular MSM - men who have sex with men, MTCT - mother to child transmission, OM-outer membrane, $P O C$ - point-of-care, STS - serological tests for syphilis, $S T I-$ sexually transmitted infection, TROMP - treponemal rare outer membrane proteins.

\section{INTRODUCTION}

Syphilis is one of seven curable, sexually transmitted infections (STIS) that is caused by a bacterial pathogen. The World Health Organization (WHO) estimates that each year 11 million new cases of syphilis occur globally among adults aged 15-49 years [1]. Syphilis remains an important public health problem in many low-income countries where it is endemic and it has re-emerged in several highincome countries, particularly in high-risk groups such as men who have sex with men (MSM) [2-4]. Cases of syphilis in MSM are a major concern because the lesions of early syphilis increase the risk of acquiring and transmitting infection with the human immunodeficiency virus (HIV) $[5,6]$ If left untreated, syphilis can cause irreversible damage to the cardiovascular and central nervous systems, resulting in morbidity and possibly mortality [7]. Furthermore, untreated early syphilis in pregnant women results in perinatal death in up to $40 \%$ of cases and can cause physical deformity and neurological complications in children who survive $[8,9]$. Control of syphilis is mainly dependent on the timely diagnosis and prompt treatment of infected individuals and their contacts with benzathine penicillin $G$ (BPG), the first-line drug for all stages of syphilis [10].

\section{ETIOLOGY OF SYPHILIS}

The etiological agent of syphilis, Treponema pallidum subsp. pallidum (T. pallidum), was identified by Schaudinn and Hoffmann in 1905 [11]. T. pallidum is an environmentally 
fragile, microaerophilic, spiral-shaped, motile bacterium. Although it has eluded continuous cultivation in vitro, it can be propagated by intratesticular infection of rabbits, the preferred animal model [12]. This spirochete is $6-20$ $\mu \mathrm{m}$ in length with a diameter of 0.1-0.2 $\mu \mathrm{m}$. Because of its narrow diameter, $T$. pallidum cannot be visualized by bright-field microscopy. However, it can be visualized with special stains (e.g., silver stain) or by phase-contrast or dark-field microscopy. The latter is an important tool for detection of $T$. pallidum in genital or cutaneous lesions of patients with early syphilis [12].

The cellular architecture of T. pallidum is similar to that of Gram-negative bacteria $[2,6,12]$. It has an outer membrane (OM), a thin peptidoglycan cell wall and an inner membrane. However, unlike Gram-negative bacteria, the $T$. pallidum flagella are located in the periplasm, and the OM, which is devoid of lipopolysaccharide (LPS), is more susceptible to disruption by mild physical manipulation and chemical treatments. As demonstrated by freeze-fracture electron microscopy, the $T$. pallidum OM contains only a few integral membrane proteins $[2,6,12]$. Because some of the treponemal rare OM proteins (TROMPs) are exposed on the cell surface, they are likely to be important during infection and are potential vaccine targets. However, the fragility of the T. pallidum OM has hindered definitive identification of the TROMPs.

The complete genome sequence of $T$. pallidum Nichols, the type strain, which was determined in 1998, has provided a wealth of information concerning the metabolism and physiology of this spirochete [13]. The Nichols genome and that of more recently sequenced strains (e.g., SS14, Chicago, Dal-1, Mexico A) is about $1.14 \mathrm{Mbp}$, encodes about 1,000 proteins, and lacks genetic elements (e.g., plasmids, bacteriophage, transposons) that are commonly associated with horizontal gene transfer $[13,14]$. The small size of the T. pallidum genome is consistent with its limited biosynthetic capabilities, resulting in a long generation time (i.e., 30-33 hours in vivo), sensitivity to various environmental conditions (e.g., oxygen, temperature, $\mathrm{pH}$ ), and dependency on the host for multiple nutrients. T. pallidum possesses a single, multigene family encoding 12 paralogous proteins, designated the $T$. pallidum repeat proteins (TprA-TprL). The function and location of these proteins has been a matter of some debate. However, the genetic sequences encoding the TprE, G, and J proteins are, for the most part, sufficiently stable within a strain, but variable among strains, to make them useful for molecular typing when combined with other genomic targets (e.g., arp and tp0548) [15-17]. The TprK protein is the most variable of the Tpr proteins with several variants typically observed within a strain as a result of a gene conversion mechanism $[6,18,19]$. Antigenic variation of the TprK protein during infection may promote chronic infection via immune evasion $[6,20]$.

\section{EPIDEMIOLOGY OF RE-EMERGENT SYPHILIS - U.S. AND CHINA}

The U.S. rate of primary and secondary syphilis, the earliest and most infectious stage, declined almost $90 \%$ during
1990-2000 [21]. The low rate of syphilis and the concentration of the majority of cases in a small number of geographic areas prompted the Centers for Disease Control (CDC) to launch a plan in 1999 to eliminate syphilis from the U.S. In 2000, the rate of primary and secondary syphilis was the lowest since reporting began in 1941 (2.1 cases per 100,000 population) $[4,21]$. However, the rate of primary and secondary syphilis increased annually during 2001-2009, decreased slightly in 2010, remained unchanged in 2011, and then increased 22\% during 20112013 to 5.3 cases per 100,000 population [4, 21]. In 2013 men accounted for $91.1 \%$ of all primary and secondary syphilis cases with the highest rates among non-Hispanic black men aged $20-29$ years [21]. MSM accounted for $75 \%$ of all primary and secondary syphilis cases. Based on available data (i.e., from 30 states and the District of Columbia), HIV co-infection was present in almost $52 \%$ of MSM with syphilis [21]. While the majority of primary and secondary syphilis cases occurred in MSM, transmission of syphilis in men who have sex with women continued in some localities and led to an increase in cases of congenital syphilis in pregnant women from 8.4 cases per 100,000 live births in 2012 to 8.7 cases per 100,000 live births in 2013 [21]. The U.S. South continued to comprise the largest proportion of cases of primary and secondary syphilis in 2013 , although the rate in the West exceeded the rate in the South. The total number of syphilis cases (all stages) reported to the CDC increased 13\% during 2012-2013 to 56, 471 cases for a rate of 18 cases per 100,000 population [21].

The most dramatic change in reported cases of syphilis in recent decades occurred in China [22-25]. Although syphilis was nearly eliminated in China during the early 1960s, cases began to rise in the 1980s. From 1991 to 2005, syphilis cases increased almost 16 -fold to $16 \%$ of the total cases of STIs. By 2008, syphilis cases had more than doubled, accounting for almost $38 \%$ of the total cases of STIs. In 2013, 444,952 cases of syphilis were reported with a rate of almost 33 cases per 100,000 population. The syphilis epidemic in China is largely driven by economic and social factors, including migration and evolving sexual mores [22-25]. MSM, female sex workers, migrants and drug users are the groups at highest risk for syphilis and for coinfection with HIV [22-25]. Rapid economic development in China has led to an unprecedented number of migrants moving from less developed rural communities to more affluent urban centers. This trend is expected to continue over the next 20 years. Many of the migrants are younger, less educated males who work in low-wage industries or service sectors. Migrants typically lack adequate health insurance, do not use medical facilities due in part to economic reasons, and cannot access social welfare benefits that are available to urban residents. This demographic poses a major public health challenge with respect to STIs because studies suggest that migrants are more likely to engage in STI-associated risk factors (e. g., low condom use, multiple sexual partners, buying and/or selling sex) [22, 25]. 


\section{CLINICAL MANIFESTATIONS OF UNTREATED ADULT SYPHILIS}

Syphilis is a chronic, systemic infection that is typically transmitted by contact with active lesions of a sexual partner (acquired syphilis) [7, 26, 27]. Syphilis can also be transmitted from an infected pregnant woman to her fetus or neonate (congenital syphilis). This STI is characterized by periods of active, clinical disease that are interrupted by periods of latency. Based on clinical findings, untreated adult syphilis can progress through four overlapping stages (i.e., primary, secondary, latent, and tertiary). The primary and secondary stages are the most infectious. Syphilis generally follows a similar course in individuals with and without HIV infection [6, 7]. (For pictures of the stages of syphilis, see reference 7.)

Primary syphilis occurs following an incubation period of about 2-6 weeks $[7,26,27]$. This stage is characterized by the presence of a painless, indurated ulcer (i.e., chancre) at the site of inoculation (typically the genital, anal or oral mucosa) and by moderate regional lymphadenopathy. In a few weeks, the chancre heals spontaneously due to phagocytosis of the treponemes by activated macrophages, which is enhanced by opsonizing antibodies [6, 27]. Although most of the treponemes are killed, some escape, possibly due to antigenic variation of the TROMPs, and disseminate to multiple organs via blood and lymph to cause chronic infection.

Secondary syphilis results from widespread multiplication of disseminated T. pallidum despite high levels of antitreponemal antibodies [7, 26, 27]. This stage, which occurs simultaneously with, or up to six months after, the healing of the chancre, is typically characterized by malaise, headache, low-grade fever, generalized lymphadenopathy, a localized or generalized skin rash with lesions on the palms and soles, mucous patches in the oral cavity or genital tract, wart-like lesions (condylomata lata), and patchy hair loss. Secondary syphilis can last for weeks or months. Relapses occur in about one-quarter of untreated patients.

Latent syphilis is the period from the disappearance of the secondary stage manifestations to the occurrence of the tertiary (late) stage manifestations [7, 26, 27]. Latent syphilis is arbitrarily divided into early latent (i.e., within one year of infection) and late latent (i.e., after one year of infection). Clinical symptoms are not apparent during latent syphilis, but serologic tests for detection of antibodies to $T$. pallidum remain positive, indicating that treponemes are still present in the lymph nodes and spleen. However, the absence of exposed mucosal lesions usually precludes venereal transmission of syphilis. About two-thirds of untreated syphilis patients remain in the latent stage for life.

Tertiary (i.e., late) syphilis, which occurred during the pre-antibiotic era in about one-third of untreated syphilis patients, is rare today due to curative antibiotics given for early syphilis or given co-incidentally for unrelated infections $[7,26,27]$. This stage usually presents within several years to a few decades after infection. Tertiary syphilis can affect almost any tissue or organ. Treponemes invade the cardiovascular and central nervous systems, eyes, skin, bones and internal organs. Damage occurs due to the invasive properties and inflammation-provoking cellular components of the treponemes that evoke a delayed-type hypersensitivity response. Manifestations of tertiary syphilis include cardiovascular syphilis, neurosyphilis, and gummatous syphilis. The later involves granulomatous lesions (gummas) that are mostly present in skin and bones, occur singly or multiply, and vary in size from microscopic to large tumor-like masses. Gummas do not usually cause physical incapacity or death, but serious complications can occur if they are present in the brain or heart. Symptoms of tertiary syphilis can include difficulty in movement coordination, paralysis, numbness, blindness, and dementia. Because so few treponemes are present during tertiary syphilis, sexual transmission of syphilis is very unlikely.

\section{CONGENITAL SYPHILIS}

Mother to child transmission (MTCT) of syphilis, commonly known as congenital syphilis, continues to affect pregnant women worldwide and is an important cause of adverse outcomes of pregnancy [27-29]. The overwhelming proportion of MTCT of syphilis occurs in pregnant women living in low-income countries. Estimates suggest that in 2008, 1.4 million pregnant women had active syphilis, which resulted in 520,000 harmful outcomes including 215,000 stillbirths, 90,000 neonatal deaths, 65,000 pre-term or low birthweight infants and 150,000 infants with congenital infection [28]. T. pallidum can be transmitted via the bloodstream of an infected pregnant woman to her developing fetus at any time during pregnancy, although the risk of fetal infection is much higher during untreated, early maternal syphilis than during late stage maternal syphilis. Congenital syphilis is divided into stages with the early manifestations appearing during the first 2 years of age and the late manifestations appearing after 2 years of age [27]. Early manifestations are infectious, resembling severe symptoms of adult secondary syphilis, and may include rhinitis, mucocutaneous lesions, osteochondritis of the long bones, hepatosplenomegaly, lymphadenopathy, anemia, jaundice and neurosyphilis. Late manifestations may include interstitial keratitis, eight-nerve deafness, interferences with tooth development, neurosyphilis, perioral fissures (rhagades), damage to long bones (sabre shins) and perforation of the nasal septum (saddle nose). MTCT of syphilis is preventable with early prenatal screening and penicillin treatment of infected pregnant women $[10,29]$. Infants born to women with untreated or inadequately treated syphilis or infants that have physical or laboratory findings that are consistent with syphilis must be treated to prevent progression of the infection [10].

\section{LABORATORY DIAGNOSIS OF SYPHILIS}

Because T. pallidum cannot be cultivated in vitro, laboratory-based diagnosis of syphilis is usually dependent upon visualization of the spirochete in clinical specimens by dark-field microscopy, which may not be available in lowincome countries, and/or reactivity of patient serum or plasma in serological tests $[12,17,26,30]$. Nucleic acid 
amplification tests, such as the polymerase chain reaction (PCR), have not been used routinely for syphilis diagnosis since a commercial test is not available. However, PCR tests for syphilis can be performed in U.S. clinical laboratories that have developed and verified their own tests in accordance with the Clinical Laboratories Improvement Amendment (CLIA) [31].

Serological tests for syphilis (STS) are traditionally divided into two types: nontreponemal and treponemal [12, $17,26,30]$. These tests measure two distinctly different kinds of antibody reactivities with different kinetics. It is important to note that the currently available STS cannot differentiate venereal syphilis from the endemic treponematoses, which are infections that are caused by the closely related $T$. pallidum subsp. pertenue (yaws) and subsp. endemicum (bejel) and T. carateum (pinta) [12, 26]. Although PCR combined with genomic sequencing can enable differentiation of the Treponema pallidum subspecies, few clinicians have access to these molecular methods [14]

Nontreponemal STS detect IgM and IgG antibodies to lipoidal antigens that are putatively released from damaged host cells $[12,26,30]$. These flocculation tests include the Venereal Disease Research Laboratory (VDRL), rapid plasma reagin (RPR), and toluidine red unheated serum test (TRUST). Nontreponemal tests are widely available, rapid, and inexpensive and can be used quantitatively to monitor the effectiveness of antibiotic treatment (i.e., defined as a 4-fold decline in RPR titer at 6-12 months after treatment). However, the use of nontreponemal STS as screening tests is problematic because they lack sensitivity in primary and tertiary syphilis. Additionally, these tests lack specificity because reactive antibodies can develop in other diseases and medical conditions that are unrelated to syphilis, resulting in biological false positives (BFPs).

Treponemal STS use native or recombinant $T$. pallidum antigens and detect IgM and IgG antibodies to treponemal components $[12,26,30]$. These tests include the T. pallidum particle agglutination assay (TPPA), the fluorescent treponemal antibody absorption assay (FTA-ABS), various enzyme-linked immunoassays (EIAs), chemiluminescence immunoassays (CIAs), and rapid point-of-care (POC) immunochromatographic strip assays. In general, treponemal STS are more sensitive and specific than nontreponemal STS for all stages of syphilis and BFPs are less common. However, because treponemal STS cannot distinguish active infection from past or treated infection, they cannot be used to monitor the effectiveness of antibiotic treatment.

U.S. guidelines for serological diagnosis of syphilis recommend the combined use of one nontreponemal STS and one treponemal STS $[12,26]$. The use of only one type of STS is insufficient for diagnosis since each type of STS has its limitations. The traditional algorithm for syphilis diagnosis is based on the use of a nontreponemal STS for screening followed by a treponemal STS for confirmation of a positive screening test. In recent years, several clinical laboratories and blood banks have switched to a "reverse algorithm" in which screening is performed with a treponemal STS followed by a nontreponemal STS when the former test is reactive $[17,26,30,32]$. However, patients with a positive treponemal STS, but a nonreactive nontreponemal STS, could have very early syphilis, longstanding latent syphilis, past treated syphilis or a BFP result. Thus, a good clinical history is essential to distinguish atrisk individuals for whom a positive treponemal STS is likely to be a true positive from individuals whose syphilis was previously treated. For individuals with no history of treated syphilis, a second, but different, treponemal STS of equivalent sensitivity should be performed. A positive second treponemal STS confirms that the patient has or had syphilis. If the second treponemal STS is nonreactive, the clinician may decide that further testing is not needed or that treatment is warranted for individuals at high-risk. There is some concern that use of the reverse algorithm could lead to increased patient follow-ups, unnecessary treatment, and higher medical costs. Several issues must be considered prior to use of this algorithm, including syphilis prevalence and the probability of a patient having syphilis based on individual risk factors.

\section{TREATMENT, PREVENTION AND CONTROL OF SYPHILIS IN ADULTS}

Because there is no vaccine to prevent syphilis, timely diagnosis and treatment of infected individuals and their sexual partners is key to syphilis control programs that also include sex education and promotion of condom use to prevent infection. A major breakthrough for syphilis treatment occurred in 1943 when Mahoney and colleagues reported the use of penicillin to successfully cure patients with primary syphilis [33]. Unlike most other bacterial pathogens that quickly developed resistance to penicillin, $T$. pallidum has remained exquisitely sensitive to this antibiotic. The U.S. CDC Sexually Transmitted Diseases (STD) Treatment Guidelines, 2015 recommend BPG as the firstline drug for incubating syphilis and for all stages of syphilis [10]. A single intramuscular (IM) injection of 2.4 million units (MU) of BPG is curative for early, uncomplicated syphilis in adults (i.e., primary, secondary, or early latent stage). An IM injection of 2.4 MU of BPG given once weekly for 3 weeks is recommended for late latent syphilis, syphilis of unknown duration and tertiary syphilis. Adults with HIV co-infection should be treated the same as those without HIV infection. Because there are no proven alternatives to penicillin for treatment of pregnant women with syphilis, those who are allergic to penicillin should be desensitized and then treated with penicillin $[10,29]$.

Data to support the use of second-line drugs for syphilis treatment consist mostly of small, uncontrolled, retrospective studies with a few larger, randomized trials [10, 26, 29]. According to the CDC STD treatment guidelines, men and non-pregnant women with early syphilis who are allergic to penicillin may be treated with doxycycline $(100 \mathrm{mg}$ orally, twice daily for 14 days) or tetracycline (500 mg orally, four times daily for 14 days) or ceftriaxone ( $1 \mathrm{~g}$ daily either IM or intravenously (IV) for 10-14 days) [10, 26]. The optimal dose and duration of the latter antibiotic have not been defined. Azithromycin ( $2 \mathrm{~g}$ orally as a single dose) 
may be used with caution, but only when treatment with penicillin or doxycycline is not feasible. Azithromycin should not be used in MSM, patients with HIV infection, or pregnant women. Careful clinical and serological follow-up of patients receiving any second-line drug is essential because treatment failure can extend the "window of opportunity" for transmission of syphilis [10, 17, 26, 29, 34].

\section{MACROLIDE-RESISTANT T. PALLIDUM}

Oral azithromycin was shown to be effective for treatment of early syphilis in non-randomized studies and in randomized controlled trials in the U.S, Africa, China, and Madagascar that compared cure rates for this macrolide and penicillin [34-39]. Azithromycin was used for syphilis treatment in Uganda (mid-1990s), in the U.S. (1999 and 2000) and in Canada (2000). However, during 2002-2003, several cases of clinical failure following azithromycin treatment were observed in syphilis patients in San Francisco, CA [40]. Laboratory analysis of samples from these patients showed the presence of an A2058G mutation in the $T$. pallidum $23 \mathrm{~S}$ rRNA gene. This mutation is identical to the $23 S$ rRNA gene mutation reported earlier by Stamm and Bergen [41] in T. pallidum SS14, a clinical isolate from a patient who failed intensive erythromycin treatment, that was shown previously to be highly resistant to erythromycin and cross-resistant to azithromycin [42]. Retrospective analysis of samples from syphilis patients in three U.S. cities (i.e., Baltimore, San Francisco, Seattle) and in Vancouver, Canada showed that although the proportion of samples containing $T$. pallidum with the A2058G mutation varied between these sites, it increased significantly over time within the sites [26, 34, 40, 43]. Macrolide-resistant T. pallidum with the A2058G mutation is now present in the U.S., Canada, Europe, China, and Australia [26, 34, 43-53]. Additionally, in 2009, Matejková et al. [44] identified a new mutation, A2059G, in syphilis patients in the Czech Republic that has subsequently been detected in syphilis patients in England, the U.S. and China [48, 52, 53]. Most T. pallidum strains containing the A2058G or A2059G mutation can be divided into multiple molecular types indicating that these mutations are not restricted to a single strain type $[51,54]$. The origin of the macrolide-resistant $T$. pallidum strains is unknown. However, Marra et al. [54] and others $[45,52]$ reported that these strains were more likely to be found in syphilis patients who had used macrolides previously for treatment of unrelated infections, suggesting that the persistence of low levels of macrolides in a patient's tissues could have selected for macrolide-resistant $T$. pallidum that arise de novo. The geographical prevalence of macrolide-resistant $T$. pallidum varies widely (e.g., $0.7 \%$ in Taiwan; nearly $100 \%$ in China) $[34,45,49,50]$. In regions where macrolide-resistant $T$. pallidum strains are now highly prevalent, these strains may be able to persist in sexual networks if patients who are infected with macrolide-resistant T. pallidum are not treated with curative antibiotics and continue to transmit the infection. Studies in the rabbit model of syphilis indicate that the T. pallidum 235 rRNA A2058G mutation is stable in the absence of se- lective pressure [34, 54]. Even in regions that currently have low levels of macrolide-resistant $T$. pallidum, the use of macrolides for syphilis treatment cannot continue without adequate surveillance due to the potential introduction of macrolide-resistant strains. Chen et al. [55] developed a real-time triplex PCR assay that can rapidly detect the $T$. pallidum A2058G and A2059G mutations. Timely identification of macrolide-resistant $T$. pallidum strains with this assay could be useful for informing physicians as to the treatment of penicillin-allergic patients with early syphilis.

\section{CONCLUSIONS}

Syphilis continues to present challenges to global public health, particularly because it increases the risk of acquiring and transmitting infection with HIV. Nonetheless, syphilis has many features of a disease that could be eliminated [56-58]. For example, syphilis only affects humans; there is no animal or environmental reservoir. Serodiagnostic tests, though not perfect, are inexpensive and widely available. Early, uncomplicated syphilis is treatable with a single IM injection of BPG, an antibiotic for which $T$. pallidum is unlikely to develop resistance [26, 34]. Finally, the 2-6 week incubation period of syphilis provides an opportunity to interrupt transmission via prophylactic treatment of sexual contacts. Despite these features, syphilis has resurged in several developed countries including China, which has recently experienced high burdens of several STIs. Host-associated factors that drive the re-emergence and spread of syphilis include high-risk sexual activity, migration and travel, and economic and social changes that limit access to health care [2, 22, 23, 25, 59].

In the absence of a vaccine, several obstacles must be addressed to enable the control of infectious syphilis. First, the spread of syphilis is dependent on the average number of new cases that are generated by an infected individual who is most contagious during the primary and secondary stages of the disease [58]. The earlier that an infected individual seeks treatment, the sooner the chain of transmission will be broken. However, the typically painless chancre of primary syphilis, which may go undetected if it is present in a less visible body area (e.g., vagina, cervix, anus/rectum), can contribute to persistence and transmission of infection, particularly if individuals do not perceive that they are at risk for syphilis. Additionally, the protean manifestations of secondary syphilis can be misdiagnosed Sexual health education campaigns targeted to high-risk groups via internet and mobile technologies are critical to raise awareness of syphilis (i.e., risk, symptoms, treatment) and to increase syphilis testing rates [60]. Healthcare providers must also be educated to recognize and treat syphilis promptly. Second, availability of simple and accurate POC tests is the key. Newer, dual target POC tests that combine nontreponemal and treponemal antigens, such as the Dual Path Platform (DPP) Syphilis Screen \& Confirm test (Chembio Diagnostic Systems Inc., Medford, NY), provide rapid results thereby allowing for same day diagnosis and treatment, which is particularly important for patients who may not return for laboratory-based test results [61- 
64]. Wider use of the POC tests is critical to expand syphilis screening to high-risk populations and to reduce MTCT of syphilis in low- and middle- income countries. Additionally, syphilis testing provides an opportunity to simultaneously screen for other STIs. Given the high rate of co-infection of syphilis and HIV, POC tests that can detect both infections have been developed and are undergoing further evaluation [65]. Third, improved contact tracing of the sexual partners of syphilis patients is crucial to enable prophylactic treatment. This can be complicated, particularly with MSM who use websites and smart phone dating apps to seek anonymous sexual partners [60]. However, internetbased strategies to address this issue have been used successfully to locate, test, and treat anonymous partners and to alert MSM to syphilis. Fourth, because of the emergence and increasing prevalence of macrolide-resistant $T$. pallidum, the use of oral azithromycin as a second-line drug for treatment of syphilis is essentially precluded $[10,26]$. Although there is as yet no documented resistance of $T$. pallidum to the tetracyclines, the other main class of second-line drugs, patient compliance is likely to be an issue $[26,34,66]$. Thus, new, single-dose oral antibiotics are needed, particularly in resource-limited settings where IM injections are problematic or for non-pregnant, penicillinallergic patients. Fifth, the majority of syphilis cases today are in individuals who typically engage in high-risk sexual behavior (e.g., MSM, commercial sex workers, migrants, drug users) and who may have limited access to health care as a result of economic issues and/or social stigmatization. Syphilis testing and treatment need to be widely available, affordable, and destigmatized to enable access by these groups. In the 1980s, changes in sexual behavior were more achievable because of the impact of HIV infection and the lack of an effective treatment. While behavioral change campaigns may not engender as great an effect today, they are still important to encourage consistent use of latex condoms and reduction in partner numbers actions that benefit the control of syphilis as well as other STIs $[59,60]$. Finally, surveillance must be strengthened to identify new syphilis outbreaks, thereby enabling a rapid response for treatment of infected individuals and their contacts, and to determine which intervention strategies are working and warrant expansion. Although eradication of syphilis may not be possible at this juncture, prior experience has shown that elimination (i.e., regional control) of syphilis is achievable provided that we make optimal use of the tools at hand and do not allow ourselves to again become complacent.

\section{CONFLICT OF INTEREST}

The author declares no conflict of interest.

\section{COPYRIGHT}

(C) 2016 Stamm. This is an open-access article released under the terms of the Creative Commons Attribution (CC BY) license, which allows the unrestricted use, distribution, and reproduction in any medium, provided the original author and source are acknowledged.

Please cite this article as: Lola V. Stamm (2016). Syphilis: Reemergence of an old foe. Microbial Cell 3(9): 363-370.

\section{REFERENCES}

1. World Health Organization (2011). Prevalence and incidence of selected sexually transmitted infections Chlamydia trachomatis, Neisseria gonorrhoeae, syphilis and Trichomonas vaginalis: methods and results used by WHO to generate 2005 estimates. World Health Organization, Geneva. Available at: http://apps.who.int/iris/bitstream/10665/44735/1/9789241502450_e ng.pdf. [accessed: 12.08.2015].

2. Stamm LV and Mudrak B (2013). Old foes, new challenges: syphilis, cholera and TB. Future Microbiol 8(2): 177-189.

3. European Centre for Disease Prevention and Control (2014). Annual epidemiological report. Sexually transmitted infections, including HIV and blood-borne viruses. Available at: www.ecdc.europa.eu. [accessed: 12.08.2015].

4. Patton ME, Su JR, Nelson R, and Weinstock H (2014). Primary and secondary syphilis- United States, 2005-2013. MMWR 63(18): 402-406.

5. Galvin SR and Cohen MS (2004). The role of sexually transmitted disease in HIV transmission. Nat Rev Microbiol 2(1): 33-42.

6. Ho EL and Lukehart SA (2011). Syphilis: using modern approaches to understand an old disease. J Clin Invest 121(12): 4584-4592.

7. Sparling PF, Swartz MN, Musher DM, and Healy BP (2008). Chapter 37. Clinical manifestations of syphilis. In: Holmes KK, Sparling PF, Stamm WE, Piot P, Wasserheit JN, Corey L, Cohen MS, Watts DH, editors. Sexually Transmitted Diseases, 4th edition. McGraw Hill, New York; pp. 661-684.

8. Ingraham NR (1951). The value of penicillin alone in the prevention and treatment of congenital syphilis. Acta Dermatol Venereol 31(Suppl 12): 60-88.

9. Schmid GP, Stoner BP, Hawkes S, and Broutet N (2007). The need and plan for global elimination of congenital syphilis. Sex Transm Dis 34(Suppl 7): S5-10.

10. Workowski KA and Bolan GA (2015). Sexually transmitted diseases treatment guidelines, 2015. MMWR Recomm Rep 64(RR-03): 1-137.

11. Waugh M (2005). The centenary of Treponema pallidum: on the discovery of Spirochaeta pallida. Int J STD AIDS 16(9): 594-595.

12. Seña AC, Pillay A, Cox DL, and Radolf JD (2015). Chap. 60. Treponema and Brachyspira, human-associated spirochetes. In: Jorgensen J, Pfaller M, Carroll K, Funke G, Landry M, Richter S, Warnock D, editors. Manual of clinical microbiology, 11th edition. ASM Press, Washington, DC; pp. 1055-1081.

13. Fraser CM, Norris SJ, Weinstock GM, White O, Sutton GG, Dodson R, Gwinn M, Hickey EK, Clayton R, Ketchum KA, Sodergren E, Hardham JM, McLeod MP, Salzberg S, Peterson J, Khalak H, Richardson D, Howell JK, Chidambaram M, Utterback T, McDonald L, Artiach P, Bowman C, Cotton MD, Fujii C, Garland S, Hatch B, Horst K, Roberts K, Sandusky M, Weidman J, Smith HO, and Venter JC. (1998). Complete genome sequence of Treponema pallidum, the syphilis spirochete. Science 281(5375): 375-388. 
14. Šmajs D, Norris SJ, and Weinstock GM (2012). Genetic diversity in Treponema pallidum: implications for pathogenesis, evolution, and molecular diagnostics of syphilis and yaws. Infect Genet Evol 12(2): 191-202.

15. Pillay A, Liu H, Chen CY, Holloway B, Sturm AW, Steiner B, and Morse SA (1998) Molecular subtyping of Treponema pallidum subspecies pallidum. Sex Transm Dis 25(8): 408-414.

16. Marra CM, Sahi SK, Tantalo LC, Gordornes C, Reid T, Behets F, Rompalo A, Klausner JD, Yin YP, Mulcahy F, Golden MR, CenturionLara A, and Lukehart SA (2010). Enhanced molecular typing of Treponema pallidum: geographical distribution of strain types and association with neurosyphilis. J Infect Dis 202(9): 1380-1388.

17. Tipple C and Taylor GP (2015). Syphilis testing, typing, and treatment follow-up: a new era for an old disease. Curr Opin Infect Dis 28(1): $53-60$

18. Stamm LV and Bergen HL (2000). The sequence-variable, singlecopy tprK gene of Treponema pallidum Nichols strain UNC and Street strain 14 encodes heterogeneous TprK proteins. Infect Immun 68(11): 6482-6486.

19. Centurion-Lara A, Lafond RA, Hevner K, Gordornes C, Molini BJ, Van Voorhis WC, and Lukehart SA (2004). Gene conversion: a mechanism for generation of heterogeneity in the tprk gene of Treponema pallidum during infection. Mol Microbiol 52(6): 1579-1596.

20. LaFond RE, Molini BJ, Van Voorhis WC, and Lukehart SA (2006). Antigenic variation of TprK $\mathrm{V}$ regions abrogates specific antibody binding in syphilis. Infect Immun 74(11): 6244-6251.

21. Centers for Disease Control and Prevention (2014). 2013 Sexually transmitted diseases surveillance. Syphilis. Available at: www.cdc.gov/std/stats13/syphilis.htm. [accessed: 12.08.2015].

22. Chen XS, Yin YP, Wang QQ, and Wang BX (2013). Historical perspective of syphilis in the past 60 years in China: eliminated, forgotten, on the return. Chin Med J 126(14): 2774-2779.

23. Chen XS, Peeling RW, Yin YP, and Mabey DC (2011). The epidemic of sexually transmitted infections in China: implications for control and future perspectives. BMC Med 9: 111.

24. Zheng N, Guo Y, Padmadas S, Wang B, and Wu Z (2014). The increase of sexually transmitted infections calls for simultaneous preventive intervention for more effectively containing HIV epidemics in China. BJOG 121(Suppl 5): 35-44.

25. Zou $X$, Chow EPF, Zhao $P, X u Y$, Ling $L$, and Zhang $L$ (2014). Ruralto-urban migrants are at high risk of sexually transmitted and viral hepatitis infections in China: a systematic review and meta-analysis. BMC Infect Dis 14: 490.

26. Stamm LV (2015). Syphilis: antibiotic treatment and resistance. Epidemiol Infect 143(8): 1567-1574.

27. LaFond RE and Lukehart SA (2006). Biological basis for syphilis. Clin Microbiol Rev 19(1): 29-49.

28. Newman L, Kamb M, Hawkes S, Gomez G, Say L, Seuc A, and Broutet $N$ (2013). Global estimates of syphilis in pregnancy and associated adverse outcomes: analysis of multinational antenatal surveillance data. PLoS Med 10(2): e1001396.

29. Clement ME, Okeke NL, and Hicks CB (2014). Treatment of syphilis: a systematic review. JAMA 312(18): 1905-17.

30. Morshed MG and Singh AE (2015). Recent trends in the serologic diagnosis of syphilis. Clin Vaccine Immunol 22(2): 137-47.

31. Council of State and Teritorial Epidemiologists (2016). Update to Public Health Reporting and National Notification for Syphilis. Available
http://c.ymcdn.com/sites/www.cste.org/resource/resmgr/PS/13-ID04.pdf. [Accessed 04.01.2016].

32. Seña AC, White BL, and Sparling PF (2010). Novel Treponema pallidum serologic tests: a paradigm shift in syphilis screening for the 21st century. Clin Infect Dis 51(6): 700-708.

33. Mahoney JF, Arnold RC, and Harris A (1943). Penicillin treatment of early syphilis - a preliminary report. Am J Public Health Nations Health 33(12): 1387-1391.

34. Stamm LV (2010). Global challenge of antibiotic-resistant Treponema pallidum. Antimicrob Agents Chemother 54(2): 583-589.

35. Ghanem KG and Workowski KA (2011). Management of adult syphilis. Clin Infect Dis 53(Suppl 3): S110-128.

36. Kiddugavu MG, Kiwanuka N, Wawer MJ, Serwadda D Sewankambo NK, Wabwire-Mangen F, Makumbi F, Li X, Reynolds SJ, Quinn TC, the Rakai Study Group, and Gray RH (2005). Effectiveness of syphilis treatment using azithromycin and/or benzathine penicillin in Rakai, Uganda. Sex Transm Dis 32(1): 1-6.

37. Hook EW III, Martin DH, Stephens J, Smith BS, and Smith K (2002). A randomized, comparative pilot study of azithromycin versus benzathine penicillin $\mathrm{G}$ for treatment of early syphilis. Sex Transm Dis 29(8): 486-490

38. Riedner G, Rusizoka M, Todd J, Maboko L, Hoelscher M, Mmbando D, Samky E, Lyamuya E, Mabey D, Grosskurth H, and Hayes R (2005). Single dose azithromycin versus penicillin $G$ benzathine for the treatment of early syphilis. N Engl J Med 353 (12): 1236-1244.

39. Hook EW III, Behets F, Van Damme K, Ravelomanana N, Leone $P$, Sena AC, Martin D, Langley C, McNeil L, and Wolff M (2010). A phase III equivalence trial of azithromycin versus penicillin for treatment of early syphilis. J Infect Dis 201(11): 1729-1735.

40. Katz KA and Klausner JD (2008). Azithromycin resistance in Treponema pallidum. Curr Opin Infect Dis 21(1): 83-91.

41. Stamm LV and Bergen HL (2000). A point mutation associated with bacterial macrolide resistance is present in both $23 \mathrm{~S}$ rRNA genes of an erythromycin-resistant Treponema pallidum clinical isolate. Antimicrob Agents Chemother 44(3): 806-807.

42. Stamm LV and Parrish EA (1990). In-vitro activity of azithromycin and CP-63,956 against Treponema pallidum. J Antimicrob Chemother 25(Suppl A): S11-14.

43. Lukehart SA, Godornes C, Molini BJ, Sonnett P, Hopkins S, Mulcahy F, Engelman J, Mitchell SJ, Rompalo AM, Marra CM, and Klausner JD (2004). Macrolide resistance in Treponema pallidum in the United States and Ireland. N Engl J Med 351: 154-158.

44. Matejková $P$, Flasarová $M$, Zákoucká $H$, Borek $M$, Kremenová $S$ Arenberger P, Woznicová V, Weinstock GM, and Šmajs D (2009). Macrolide treatment failure in a case of secondary syphilis: a novel A2059G mutation in 23S rRNA gene of Treponema pallidum subsp. pallidum. J Med Microbiol 58(Pt 6): 832-836.

45. Chen XS, Yin YP, Wei WH, Wang HC, Peng RR, Zheng HP, Zhang JP, Zhu BY, Liu QZ, and Huang SJ (2013). High prevalence of azithromycin resistance in Treponema pallidum in geographically different areas of China. Clin Microbiol Infect 19(10): 975-979.

46. Muldoon EG, Walsh A, Crowley B, and Mulcahy F (2012) Treponema pallidum azithromycin resistance in Dublin, Ireland. Sex Transm Dis 39(10): 784-786

47. Read P, Jeoffreys N, Tagg K, Guy RJ, Gilbert GL, and Donovan B (2014). Azithromycin-resistant syphilis-causing strains in Sydney, Australia: prevalence and risk factors. J Clin Microbiol 52(8): 2776 2781. 
48. Tipple C, McClure MO, and Taylor GP (2011). High prevalence of macrolide resistant Treponema pallidum strains in a London centre. Sex Transm Infect 87(6): 486-488.

49. Wu BR, Yang CJ, Tsai MS, Lee $K Y$, Lee NY, Huang WC, Wu $\mathrm{H}$, Lee $\mathrm{CH}$ Chen TC, Ko WC, Lin HH, Lu PL, Chen YH, Liu WC, Yang SP, Wu PY, Su YC, Hung CC, and Chang SY (2014). Multicentre surveillance of prevalence of the 23S rRNA A2058G and A2059G point mutations and molecular subtypes of Treponema pallidum in Taiwan, 2009-2013. Clin Microbiol Infect. 20(8): 802-807.

50. Wu H, Chang SY, Lee NY, Huang WC, Wu BR, Yang CJ, Liang SH, Lee $\mathrm{CH}$, Ko WC, Lin HH, Chen YH, Liu WC, Su YC, Hsieh CY, Wu PY and Hung CC (2012). Evaluation of macrolide resistance and enhanced molecular typing of Treponema pallidum in patients with syphilis in Taiwan: a prospective multicenter study. J Clin Microbiol 50(7): 2299-2304.

51. The A2058G Prevalence Workgroup (2012). Prevalence of the 235 rRNA A2058G point mutation and molecular subtypes in Treponema pallidum in the United States, 2007-2009. Sex Transm Dis 39(10): 794798.

52. Grimes M, Sahi SK, Godornes BC, Tantalo LC, Roberts N, Bostick D, Marra CM, and Lukehart SA (2012). Two mutations associated with macrolide resistance in Treponema pallidum: increasing prevalence and correlation with molecular strain type in Seattle, Washington. Sex Transm Dis 39(12): 954-958.

53. Li Z, Hou J, Zheng R, Li Z, Wen J, Liu D, Liu R, Chu T, Liu B, Yu G, Tian $H$, and Zhang $F$ (2013). Two mutations associated with macrolide resistance in Treponema pallidum in Shandong, China. J Clin Microbiol 51(12): 4270-4271.

54. Marra CM, Colina AP, Godornes C, Tantalo LC, Puray M, CenturionLara A, and Lukehart SA (2006). Antibiotic selection may contribute to increases in macrolide-resistant Treponema pallidum. J Infect Dis 194(12): 1771-1773.

55. Chen $\mathrm{CY}$, Chi KH, Pillay A, Nachamkin E, Su JR, and Ballard RC (2013). Detection of the A2058G and A2059G 23S rRNA gene point mutations associated with azithromycin resistance in Treponema pallidum by use of a TaqMan real-time multiplex PCR assay. J Clin Microbiol 51(3): 908-13

56. Rompalo AM (2001). Can syphilis be eradicated from the world? Curr Opin Infect Dis 14(1): 41-44.
57. Cameron CE and Lukehart SA (2014). Current status of syphilis vaccine development: need, challenges, prospects. Vaccine 32(14): 1602-1609.

58. Garnett GP and Brunham RC (1999). Magic bullets need accurate guns--syphilis eradication, elimination, and control. Microbes Infect 1(5): $395-404$

59. Althouse BM and Hébert-Dufresne L (2014). Epidemic cycles driven by host behaviour. J R Soc Interface 11(99): 20140575.

60. Drummond F, Guy R, Kaldor JM, and Donovan B (2010). The intersection between HIV and syphilis in men who have sex with men: some fresh perspectives. HIV Ther 4(6): 661-673.

61. Castro AR, Esfandiari J, Kumar S, Ashton M, Kikkert SE, Park MM, and Ballard RC (2010). Novel point-of-care test for simultaneous detection of nontreponemal and treponemal antibodies in patients with syphilis. J Clin Microbiol 48(12): 4615-9.

62. Yin YP, Chen XS, Wei WH, Gong KL, Cao WL, Yong G, Feng L, Huang SJ, Wang DM, Han Y, Chen SC, Mabey D, and Peeling RW (2013). A dual point-of-care test shows good performance in simultaneously detecting nontreponemal and treponemal antibodies in patients with syphilis: a multisite evaluation study in China. Clin Infect Dis 56(5): 659-65

63. Causer LM, Kaldor JM, Conway DP, Leslie DE, Denham I, Karapanagiotidis T, Ryan C, Wand $\mathrm{H}$, Anderson DA, Robertson PW McNulty AM, Donovan B, Fairley CK, and Guy RJ (2015). An evaluation of a novel dual treponemal/nontreponemal point-of-care test for syphilis as a tool to distinguish active from past treated infection. Clin Infect Dis 61(2): 184-191.

64. Kay NS, Peeling RW, and Mabey DC (2014). State of the art syphilis diagnostics: rapid point-of-care tests. Expert Rev Anti Infect Ther 12(1): 63-74.

65. Hess KL, Fisher DG, and Reynolds GL (2014). Sensitivity and specificity of point-of-care rapid combination syphilis-HIV-HCV tests. PLoS One 9(11): e112190.

66. Wu BR, Liu WC, Wu PY, Su YC, Yang SP, Hung CC, and Chang SY (2014). Surveillance study of Treponema pallidum harbouring tetracycline resistance mutations in patients with syphilis. Int J Antimicrob Agents 44(4): 370-372. 